\title{
Entrevista a Rossana Reguillo: La violencia del dato
}

\begin{abstract}
¿Cómo definirías el campo de la investigación en comunicaciones ahora que mucha de ella se ha confundido con asesorías y controles de flujos informáticos?
\end{abstract}

Me parece difícil generalizar una respuesta, porque encuentro que así como hay una fuerte tendencia en el campo a vincularse a la lógica del mercado, me refiero a las asesorías y al sentido pragmático en la investigación; hay también un importante espacio de trabajo crítico que busca su autonomía y estar a la altura de los desafíos que plantea la sociedad. Encuentro que el momento actual en el campo de la investigación en comunicación es muy intenso y productivo. Hay además dos tensiones palpables en su configuración: de un lado, la que representa la diversificación de objetos, enfoques y modos de entender lo comunicacional en la teoría, esto me parece sano y de hecho lo considero una fortaleza, sin embargo, la diversificación se traduce muchas veces en dispersión y ésta a su vez en disputas por la "definición legítima" de lo que es el campo. Quiero decir que la diversidad no ha logrado aún convertirse en un "valor" o, planteado en otros términos, tengo la impresión de que las diferencias de enfoques, el interés por objetos de estudio diferenciados, la búsqueda interdisciplinaria, no logran configurar un campo con subcampos. Cuando acercamientos divergentes a la centralidad de los medios aparecen en escena, hay una tendencia (todavía presente) a negar que estos acercamientos formen parte del campo de investigación en comunicación. Hay una pugna más de orden político que epistemológico, pero esto va cambiando y hoy algunos "subcampos" como la sociología de la comunicación, la antropología de la comunicación, la comunicación política, entre otros acercamientos empiezan a generar su propia acumulación de saberes y procedimientos, sin amenazar -en términos de Lakatos- el núcleo central de la comunicación. Pero este debate no está aún planteado. De otro lado, otra tensión clave del momento, la encuentro en la creciente autonomización del campo de investigación con respecto a la docencia y profesionalización de la comunicación. El tema es complejo, porque requiere historizar la emergencia del campo, pero en aras de la brevedad, lo que puedo señalar, es que encuentro que la producción de conocimiento en comunicación -en sus diversidades aún precarias- toma distancia de la lógica que la ha caracterizado por mucho tiempo: una investigación vinculada a los problemas de la profesionalización. Incluso, espacios institucionalizados tan importantes como FELAFACS, han venido abriendo espacios cada vez más visibles para colocar el problema del estudio y comprensión de la comunicación desde una lógica no necesariamente vinculada a la resolución de problemas curriculares en las infinidad de escuelas de comunicación del continente. Aquí el tema, me parece es como establecer las diferencias y al mismo tiempo las articulaciones, entre el inevitable asunto de la profesionalización, el estatuto epistemológico de los saberes comunicacionales y el proyecto político de la comunicación. Son tres aristas que hacen parte de cualquier intento de "mapear" la constitución y las transformaciones del campo.

Soy optimista, mi experiencia y contacto con diversas comunidades del campo, me llevan a pensar que estamos cada vez en mejores condiciones de enfrentar de manera articulada pero atendiendo la diferencia de problemáticas, la necesaria distinción entre profesionalización y producción científica de conocimientos. Las nuevas generaciones son portadoras de un conjunto de preguntas que desafían al campo, al tiempo que están forzando (en el buen sentido) sus límites epistemológicos.

¿Se ha perdido la dimensión política y crítica y, a cambio, se ha entronizado una especie de neofuncionalismo acorde con nuevas impunidades materiales y simbólicas?

Me considero una buena lectora de la producción en las áreas de mi interés, así como una seguidora atenta de los debates claves y no tengo evidencias del "adelgazamiento" crítico desde la comunicación. Por el contrario, encuentro una actitud combativa y una densidad crítica que me parecen lejos de las complicidades con los órdenes vigentes. Lo que me parece 
un problema serio, es la falta de espacios o de accesibilidad a los debates públicos por parte de la comunidad de estudiosos de la comunicación -sin generalizar-, las atmósferas mediáticas están impregnadas de un cierto pragmatismo donde la voz del "experto" pierde peso o es usada como contrapunteo a los relatos con cierta dosis de consagración. Pero me parece que esta situación no puede resolverse mediante un "decreto" (ahora todos buscamos ocupar un espacio en la esfera pública para hacer circular las voces críticas!); el fortalecimiento de lo que tú llamas "neofuncionalismo" y que yo llamaría, de manera tal vez simplificadora, el éxito de los chamanes y agoreros del mercado, cuya eficacia no puede atribuirse a la "pérdida de la dimensión política y crítica" de los estudios de la comunicación en particular- y de las ciencias sociales y las humanidades, en lo general-, sino a la asimetría en las condiciones de enunciación. En otras palabras, la pelea no es pareja y hay enormes dificultades para hacer circular las versiones y visiones divergentes de una realidad que se quisiera a-problemática. No, no se ha perdido la dimensión crítica, pienso, pero se han transformado los espacios y las retóricas para dar la pelea. Voy a atreverme a hacer una generalización peligrosa: la prensa, cierta prensa, los medios, algunos medios, nos necesitan como justificación de algunos de los excesos "nefuncionalistas", pero hay una fuerte tendencia a colocar la voz crítica como "pie de página". Los estudiosos, investigadores, analistas, intelectuales, no somos los epicentros de los debates, somos un coro disidente y en condiciones poco ventajosas. A veces, confieso en voz baja, me asalta una sonrisa irónica, al ver que la experiencia de los estudiosos de la comunicación y la cultura hoy, se acerca a lo que las mujeres hemos experimentado de manera histórica: la condena a la nota de pie de página y que reaparece luego como una "acusación" de falta de interés o de densidad política. Hay mucho por hacer en esta dimensión, pero repito mi visión optimista. Poco a poco, los estudiosos de la comunicación ganan en legitimidad y en espacios críticos, mi pesimismo proviene de la impresión de que el intelectual público se desdibuja. Pero nadie dijo que esta lucha era sencilla, o ¿sí?.

¿De qué manera las violencias intempestivas y las programadas están cambiando la agenda de los medios?

Este es un tema sumamente complejo y de una amplitud considerable. Las violencias se han convertido en el texto fuerte de una realidad debilitada. Me preocupan las respuestas contundentes y unívocas, por lo que me atrevería a decir que hay una interrelación entre la agenda de los medios y la visibilidad de las violencias, es decir, no se trata sólo de que la crisis en la sociedad contemporánea configure la agenda de los medios, sino también de señalar la influencia de los medios en la crisis contemporánea. A mi juicio ( y con el aval de varios años de investigación empírica sobre el tema), los medios se han transformado en actores de peso completo en la producción y reproducción del acontecer, de la realidad y en nuevos espacios de gestión de la creencia. Las violencias "legítimas", las violencias sociales e informes, alimentan el ángulo "caliente y novedoso" de los medios, pero simultáneamente se convierten en lamento y constatación de la dislocación de un proyecto que se creyó a salvo de la irrupción de la irracionalidad. Es fascinante, como objeto de estudio la relación entre medios y violencia no deja nunca de sorprenderme porque el texto de la violencia ha sido convertido en una -casi exclusiva- narrativa de la disolución social y pocas veces en los relatos mediáticos, aparece la crítica al proyecto de la modernidad y sus figuras mercadológicas. No quiero abusar de la palabra, pero el tema implicaría en primer término una problematización y redefinición de lo que las sociedades hoy, consideran "violento". En mi propia investigación he podido seguir el tránsito y las transformaciones de aquello a lo que se le atribuye la calidad de violento y encuentro una cuestión clave, no resuelta aún, entre una "mayor sensibilidad" al tema en el momento actual y al mismo tiempo una mayor capacidad o disposición para exponerse a estos asuntos. La respuesta a la pregunta concreta es, sí, las violencias reconfiguran la agenda de los medios, pero debería añadir que los medios reconfiguran la percepción sobre las violencias. Esta respuesta, requeriría citas, documentación y argumentación empíricas. De manera sintética, puedo decir que he ido estableciendo diferencias entre las violencias "legítimas" (aquellas ejercidas por el Estado y sus instituciones), las violencias históricas (las que suponen el retorno de problemas no resueltos en la sociedad) y las que llamo las violencias disciplinantes (las que constituyen un conjunto de saberes y procedimientos de control y auto-vigilancia del cuerpo social), mi hipótesis interpretativa es que los medios están mucho 
más interesados en las violencias disciplinantes ( sí, es una pena, lo mataron pero era un delincuente; pues sí, es terrible, la asesinaron y torturaron, pero era una prostituta!). Por razones complejas que no tengo oportunidad de desarrollar aquí, me parece que hay una fuerte necesidad de encontrar y de hacer circular relatos explicativos sobre lo que sucede: las violencias disciplinantes son fundamentales en esta operación. (Remito al lector interesado en estos planteamientos a mi trabajo sobre las violencias que puede ubicarse en Internet a través de: http://www.utexas.edu/cola/llilas/events/oldevents/culturaypaz/reguillo.pdf)

\section{¿Cuáles serían las relaciones que se establecen entre las formas de disolución de lo público y la comunicación?}

Tengo la impresión de que este debate comenzó pero no alcanzó conductos ni espacios de visibilización adecuados. Forma, con otro conjunto de temas, áreas claves que solemos dar por debatidas pero que en realidad aparecen y desaparecen en nuestras discusiones. Martín Barbero, García Canclini, entre otros, hicieron importantes aportaciones para pensar esta cuestión la transformación de lo público y en sus trabajos hay pistas claves para colocar en una perspectiva no apocalíptica la reconfiguración del espacio público. Hacia comienzos de la década de los noventa ¡del siglo pasado!, hubo una fuerte tendencia en varios de los estudiosos del tema a dar por sentado que los medios o, desde otra perspectiva, la tecnologización creciente de la comunicación y la transformación en los modos de "consumo", operaban de manera irreversible una "disolución de lo público"; el muy importante libro de Sennet sobre "el declive del hombre público", introdujo cuestiones fundamentales al debate. Los acontecimientos y el movimiento social, vinieron a señalar que lo público se transformaba pero cobraba nuevo vigor y otras tonalidades expresivas. En los últimos años, la recuperación del signo político de la calle (pienso en el Perú de la caída de Fujimori, en la Argentina cacerolera y piquetera, en el México zapatista, entre otro conjunto de importantes signos) ha obligado a revisar nuestras concepciones de lo público. Pero de otro lado, hay que ser cuidadosos en el análisis y reconocer que la vigorización del espacio público (el mejor ejemplo lo constituye el movimiento global anti-guerra, que detonó una "conversación" planetaria muy intensa a través de distintos mecanismos, en el 2003), no se traduce necesariamente en un fortalecimiento de la dimensión política y de agencia en lo público. La pregunta requiere colocarse, necesariamente, con referencia a sus anclajes empíricos y de manera fundamental, asumir que la configuración de lo público es siempre resultado de complejos y multidimensionales procesos tanto históricos como situacionales. Aquí habría que hacer la historia cultural de los medios, de la configuración de los Estados-nacionales, de la tradición de las sociedades locales, de las formas particulares del ejercicio del poder y de la violencia. En fin lo que me parece es que este es un debate abierto y fundamental para comprender las relaciones, las imposiciones, las resistencias que hoy aparecen, en esta atmósfera de control policiaco planetario.

¿Promueve la globalidad un tipo de imaginario mundial donde la cultura reemplaza a lo social y, al mismo tiempo, debilita la fuerza de lo político?

Si capto bien el sentido de la pregunta, me permitiría organizar mi respuesta en dos partes. De un lado, es indudable que la globalidad (en su sentido cultural), -Ortíz diría que nos referimos más a mundialización-, promueve imaginarios de distintos tipos que tienden a desplazar lo socio-político en aras de una comprensión cultural del mundo. Ello no es necesariamente malo, ni intrínsecamente bueno, porque el imaginario no es un ente que flote en alguna parte de la realidad, sino un proceso cuya fuerza expansiva depende de los anclajes que logre construir y de su capacidad para constituirse en "relato" compartido. "Imaginarios globales, anclajes locales", titulé un artículo que escribí en el 98, en el que trataba precisamente de analizar (empíricamente) los modos en que estos imaginarios de vocación global se enfrentaban a procesos de apropiación, negociación y resistencia locales. Encontré, por ejemplo, que al imaginario expandido de la democratización y la interculturalidad, las dos grandes narrativas "salvíficas" que desde la perspectiva de ciertos poderes, pretendían resuelto el problema político de las sociedades latinoamericanas, se oponía la historia de autoritarismos, recelos, racismos velados, en ciudades como Guadalajara, Medellín, San Juan en Puerto Rico, La Plata en Argentina; y en sentido contrario, a un cierto imaginario que 
construye la identidad latinoamericana como anomalía e incomplitud (con referencia a las identidades metropolitanas), se oponían estrategias de resistencia que negaban valor a este imaginario. Pienso que la perspectiva de Gramsci da para entender estos fenómenos contemporáneos de una manera creativa y rigurosa. De otro lado, habría que celebrar (yo lo celebro) lo que algunos analistas, Norbert Lechner de manera destacada, han llamado la "culturalización de la política". Es decir, no comparto la idea de que la cultura des-densifique lo político, sino que la cultura ha venido a revitalizar el ejercicio de una política demasiado acostumbrada a no abrir en sus espacios y agendas, temas cruciales para la vida social. Desde las feministas que con gran tino señalaron que "lo privado es político", hasta los nuevos movimientos sociales, como el de los jóvenes, la irrupción de prácticas, creencias, emociones, han permitido la entrada en el debate de asuntos que se escapaban por las retículas de las grandes instituciones, como diría Beck. Sin embargo, es importante reconocer que efectivamente se instala una tendencia a lo que algunos llaman el "pensamiento único" y que esta pugna será larga.

¿Los jóvenes se ha convertido en un expediente mediático del miedo, son algo a sí como los nuevos bárbaros con los que se ejemplifica la necesidad de seguridad y represión?

Sí, los jóvenes han sido convertidos en relato expiatorio y en el "enemigo" del orden social. Los medios, tanto los electrónicos como la prensa, en términos generales, han venido satanizando a los jóvenes, pero no a los jóvenes como categoría universal (que no existe), sino a ciertos jóvenes, a los jóvenes pobres en concreto. La documentación empírica sobre estos procesos de estigmatización que han convertido en sinónimo "jóvenes y peligrosidad o amenaza", son abundantes. Pero no se trata sólo del discurso de los medios de comunicación, hay que observar analíticamente también la obsesión legislativa de numerosos congresos en distintos países para la reducción de la edad penal y la creación de la figura jurídica del "delincuente juvenil"; las acciones punitivas de los gobiernos municipales que persiguen a los jóvenes, el desconcierto del sistema educativo que no logra resolver el temor (y la impotencia) que le provoca el desencanto y el cinismo de buena parte de los jóvenes. Los dispositivos de estigmatización se fortalecen en diversos territorios y contribuyen a generar una atmósfera densa, en la que los tambores de guerra retumban con signos ominosos.

La impunidad con la que las noticias, reportajes, artículos, documentales, construyen a los jóvenes como operadores y culpables de las violencias que habitan la sociedad, es indignante. A manera de "anécdota" déjame contarte que recientemente un periodista catalán me emplazaba a contestarle, en una frase sintética, cuál era el problema fundamental para los jóvenes en América Latina; supongo que esperaba que mi respuesta se inscribiera en la línea de ese imaginario de violencias desbordadas que alimenta las mitologías europeas sobre Latinoamérica, ya que no pudo ocultar su decepción cuando le contesté que los Estados latinoamericanos gastan más en el servicio de su deuda exterior que lo que invierten en política social. Pese a que la frase fue citada en la prensa, tengo la impresión de que no se ajustaba al "tropicalismo" con el que desde esa zona de la geografía suelen observarnos. Pese a la falta de "impacto mediático", estoy (sigo) convencida de que el gran problema de nuestras sociedades pasa por ahí, por Estados que han decidido (los gobernantes afirman que se han visto obligados) sacrificar a la población más vulnerable en consonancia con un proyecto mundial cuya fuerza deriva de su capacidad para producir un imaginario de fatalidad y de univocidad sin contra-relatos. Los datos documentan el pesimismo, diría Monsiváis. En la geografía latinoamericana y caribeña, donde viven alrededor de 494 millones de personas, de las cuales el 32.3\% es menor de quince años, resulta difícil entender que por ejemplo México, en 1999, invirtiera el $8.3 \%$ de su Producto Interno Bruto (PIB) al servicio de la deuda y sólo el $4.9 \%$ en educación; o que en el Brasil el 9\% del PIB se fuera en la deuda exterior y apenas el $2.9 \%$ en salud; en Ecuador mientras en 1999 se invertía 1.7 \% del PIB en salud y $3.5 \%$ en educación, ese país gastaba 8.7\% al servicio de su deuda, según datos del PNUD en el 2001. Pero alguien tiene que pagar los platos rotos, y los jóvenes se convierten en los protagonistas de una biopolítica que ciertamente se fortalece desde la retórica de los medios.

¿Es posible suponer un horizonte asimétrico donde la comunicación pueda cumplir algunas tareas de descentramiento de los discursos hegemónicos? 
No. Soy optimista, pero no tanto. Vuelvo a separar la pregunta en dos partes. Los horizontes asimétricos no existen, pensar que sí, es volver a ciertas viejas concepciones del poder, al que se pensaba como un síndrome, con Foucault aprendimos que el poder es una relación, una fuerza de múltiples direcciones y que no puede no existir. Lo que me parece delicado del momento actual es la ausencia de contrapoderes o dicho en otras palabras de contrapesos visibles a los poderes políticos, económicos, culturales. Tomemos como ejemplo la Patriot Act y la Total Information Awarness (TIA), en los Estados Unidos, instrumentos perversos que lanzan toda la fuerza del Estado sobre las libertades civiles (el artículo que a propósito de esto sacó The Guardian en noviembre del 2002, es notable como pieza informativa y espeluznante como relato), ni la Cámara de Representantes colocó un veto a este indudable abuso de poder, intelectuales críticos en los Estados Unidos, que los hay, están dando una pelea en condiciones muy adversas, están sitiados por los grandes consorcios comunicativos. El problema fundamental, me parece, es la falta de contrapesos al poder creciente del equipo

Bush. Por ello considero que efectivamente la comunicación como proceso y práctica, tiene una tarea fundamental en lo que llamas el descentramiento de los discursos hegemónicos. Abrir espacios para el debate, problematizar y cuestionar al poder, fomentar la circulación de contra-relatos, son tareas claves, en el contexto de las escandalosas asimetrías en el mundo contemporáneo. Por eso, la comunicación se ha convertido en una agenda crucial para los proyectos en disputa.

\section{¿Crees que el dilema actual está en nacionalizar la globalización para salvar lo particular de convertirse en la moda de la diferencia?}

No sé, no tengo una respuesta formulada. Depende mucho de lo que se entienda por "nacionalizar"; me preocupa por ejemplo el resurgimiento de ciertos nacionalismos que lejos de representar una solución a los problemas que nos habitan, terminan por agudizar los particularismos que impiden la interacción entre formas y procesos culturales diversos. Pero de otro lado, de manera inevitable la globalización enfrenta siempre procesos de apropiación y resemantización nacionales. Creo que desde los estudios de la comunicación hoy estamos en mejores condiciones para entender que lo global no anula lo nacional, lo reformula.

\section{¿Hay nuevos miedos?}

Sí y no. Tratemos de ubicar el significado de "nuevo", si se le comprende como algo completamente inédito habría que decir que ninguno de los miedos que están presentes en la sociedad contemporánea es nuevo en tanto cada uno de ellos se entrelaza a constantes antropológicas, a tradiciones, a relatos fundacionales, aún los miedos vinculados a la ciencia y a la tecnología están alimentados por dos miedos básicos: la criatura que se vuelve contra su creador (por ejemplo Mary Shelley dió cuenta de esto en su magnífico Frankestein) es un tema que ha acompañado la historia de la humanidad; el temor a la violación del precepto y el saber sagrados (que la modernidad no logró erradicar), acompañan la expresión de los miedos frente al avance de la ciencia y la tecnología. Sin embargo, para entender los miedos como emergencia contemporánea, me ha sido de gran utilidad la elaboración que hace Lawrence Grossberg, del concepto de articulación. El dice que se trata de la "construcción de un conjunto de relaciones a partir de otra; muchas veces supone desarticular unas relaciones con el fin de rearticular otras. La articulación es una lucha continua por resituar prácticas dentro de un campo de fuerzas cambiante, por redefinir las posibilidades de vida redefiniendo el campo de relaciones -el contexto- dentro del cual se localiza una práctica". En este sentido sí podemos hablar de nuevos miedos, en la medida en que las articulaciones que se producen resitúan nuestra comprensión, representación y discursivización de los miedos.

\section{¿Cuáles serían?}

Estoy ahora en la fase de escritura de un libro sobre la construcción social del miedo desde una perspectiva latinoamericana, investigación que me ha llevado cinco años de trabajo. Puedo decirte que hoy el miedo se instaura como condición de época, como atmósfera cultural y como proyecto político, instalando dos certezas básicas: "nadie está a salvo", "todos 
somos sospechosos". Dos consignas que operan como claves expandidas de intelección del mundo y que no sólo contribuyen a la erosión del tejido social sino que además funcionan como "coartadas" en dos direcciones: "desde arriba" justifican y celebran el autoritarismo, los discursos y las prácticas de vigilancia y control, hoy globalizadas, que han encontrado en el desencanto, en la vulnerabilidad y en el temor hacia el futuro, una filón para imponer un modelo que no acepta contestación alguna; "desde abajo", operan como ratificación de lo que los teóricos de la modernidad tardía denominan "inadecuación biográfica del yo", cuya traducción es a la vez compleja y muy elemental: todos estamos mal y no hay espacios de escapatoria ("el mundo nunca fue tan pequeño como hoy ", diría lanni), ni prácticas a prueba de la asunción de estas "verdades". Nadie está a salvo, todos somos sospechosos (siempre los otros, más que yo), son hipótesis subyacentes al modo en que nos colocamos en el mundo. Llenos de consecuencias es aquí que estamos, desvelados por la incertidumbre del presente y los contornos nebulosos de un futuro inasible, siempre en fuga.

¿Es la comunicación un espacio desde el cual podemos seguir ejerciendo una labor intelectual no cosificada que ayude a entender la crisis de representación y el escepticismo que la sostiene?

Quiero creer que sí, que la comunicación es un espacio en el sentido fuerte y antropológico que De Certeau le dio a este concepto como "lugar significado", ello quiere decir que se requiere de la existencia de un conjunto de "practicantes" dispuestos a construir y a mantener condiciones de enunciación para la palabra pública y la palabra crítica. Estamos en una severa crisis de lo que me gusta llamar la "nomenclatura" para entender el mundo, muchos conceptos están vaciados de sentido o no alcanzan a dar cuenta de lo que sucede, en ese sentido estamos ante una oportunidad excelente para poner a funcionar la creatividad con rigor y el rigor con imaginación. Me alienta siempre el debate con los colegas porque encuentro en buena parte del campo, un trabajo de la imaginación muy fecundo. El escepticismo es sin embargo, un antídoto contra los entusiasmos excesivos.

\footnotetext{
* Rossana Reguillo es profesora-investigadora del Departamento de Estudios Socioculturales del ITESO.
} 